\title{
Temperature Forecast Using Ridge Regression as Model Output Statistics
}

\author{
Niswatul Qona'ah ${ }^{1, *}$, Sutikno $^{2, * *}$, Kiki Ferawati ${ }^{1, * * *}$, Muhammad Bayu Nirwana ${ }^{1, * * * *}$ \\ ${ }^{1}$ Statistics Study Program, Faculty of Mathematics and Natural Science, Universitas Sebelas Maret, \\ Jl. Ir Sutami No.36 A, Surakarta, Jawa Tengah 57126. Tel. (+62)271-646994, Fax. (+62)271-636268. \\ ${ }^{2}$ Statistics Department, Faculty of Mathematics Computing and Data Science, Institut Teknologi Sepuluh Nopember, \\ Jl. Teknik Kimia, Keputih, Sukolilo, Surabaya, Jawa Timur 60111. \\ Email: niswatulqonaah@staff.uns.ac.id*, sutikno@statistika.ac.id**, kferawati@ statistika.ac.id***, mbnirwana@staff.uns.ac.id****
}

\begin{abstract}
Over the past few years, BMKG (Meteorological, Climatological and Geophysical Agency) in Indonesia has used numerical weather forecasting techniques, namely Numerical Weather Prediction (NWP). However, the NWP forecast still has a high bias because it is only measured on a global scale and unable to capture the dynamics of atmosphere (Wilks, 2007). Hence, this study implements Ridge Regression as Model Output Statistics (MOS) for temperature forecast. This study uses the maximum temperature (Tmax) and minimum temperature (Tmin) observation at 4 stations in Indonesia as the response variables and NWP as the predictor variable. The results show that the performance of the model based on Root Mean Square Error of Prediction (RMSEP) is considered to be good and intermediate. The RMSEP for Tmax in all stations is intermediate (0.9-1.2), Tmin in all stations is good (0.5-0.8). The prediction result from Ridge Regression is more accurate than the NWP model and able to correct up to $90.49 \%$ of the biased NWP for Tmax forecasting.
\end{abstract}

Keywords: MOS, NWP, Ridge Regression, Temperature

\section{INTRODUCTION}

Indonesia is an archipelagic country that has a tropical climate, dynamic and complex weather. Weather is considered as an inseparable part of human activities and affects various fields of life. Hence, efficient methods are needed for weather forecasting, especially in shortterm forecasting. The Indonesian Meteorology, Climatology and Geophysics Agency (BMKG) has forecasted short-term weather by comparing and observing weather patterns and conditions that occurred the day before, and in general, the accuracy of the forecast will vary because it depends on the experience of the weather forecaster (Sutikno, Nisaa' and Nur 'Anisa', 2019).

One of the obligation of the BMKG is to provide information about the weather forecast including the maximum temperature (Tmax) and minimum temperature (Tmin). BMKG has also been conducting research for short-term weather forecasts using Numerical Weather Prediction (NWP) since 2004. However, the results of the NWP forecast are still biased for locations that have complex high-resolution topography and vegetation. Hence, the Model Output Statistics (MOS) is needed to optimize the utilization of NWP outputs to produce more accurate weather forecasts (Glahn and Lowry, 1972).

MOS is a method for modeling the relationship between weather observations and NWP output based on the regression method. MOS will determine the statistical relationship between NWP as a predictor variable and response variable at some projection times
(Glahn and Lowry, 1972). The predictor variables of this study are NWP outputs which is potentially correlate with each other, for example relative humidity is potentially correlate with precipitation. Hence, ridge regression is used as the MOS method. Ridge regression is one of the methods used to reduce the effect of correlated predictors (Draper and Smith, 1998).

In this study, the response variables are the observation maximum temperature (Tmax) and minimum temperature (Tmin), while the predictor variable are the outputs data of the Numerical Weather Prediction Conformal Cubic Atmospheric Model (NWP CCAM). The NWP data is measured from 9 grids for every variable so that the complexity will be high and the multicollinearity is very likely to occur. In order to overcome the multicollinearity problem and reduce the dimensions of the grid, a PCA (Principal Component Analysis) process is carried out. The principal component produced is used as a predictor variable for ridge regression. Then, the results of ridge regression with PCA as the pre-processing stage will be compared with actual data and NWP models by looking at the RMSEP (Root-Mean-Square-Error Prediction) and\% IM criteria (percentage increase).

Section 2 describe the Principal Component Analysis (PCA) method, MOS Modeling using ridge regression, variables used, and model evaluation. The Application of the method for temperature forecasting and the results of the analysis presented in section 3. Finally, section 4 presents the conclusion of this study. This study uses statistical approach to explain about temperature forecast. 


\section{MATERIALS AND METHODS}

\section{Principal Component Analysis (PCA)}

Principal Component Analysis (PCA) is a method used to explain the structure of variance-covariance of many variables based on several linear combinations that are formed (Johnson and Wichern, 2007). PCA is generally used to reduce data with many correlated variables in order to facilitate interpretation. This is done by transforming the original variables into several principal components (PC), which are expected to no longer correlate with each other.

If a random vector $\mathbf{x}^{\prime}=\left[\begin{array}{llll}x_{1} & x_{2} & \ldots & x_{p}\end{array}\right]$ has a covariance matrix of $\Sigma$ with the eigenvalue of $\lambda_{1} \geq \lambda_{2} \geq \ldots \geq \lambda_{p} \geq 0$, then the linear combination will be in (1).

$$
\begin{aligned}
& P C_{1}=\mathbf{e}_{1}^{\prime} \mathbf{x}=e_{11} x_{1}+e_{12} x_{2}+\ldots+e_{1 p} x_{p} \\
& P C_{2}=\mathbf{e}_{2}^{\prime} \mathbf{x}=e_{21} x_{1}+e_{22} x_{2}+\ldots+e_{2 p} x_{p} \\
& \vdots \\
& P C_{p}=\mathbf{e}_{p}^{\prime} \mathbf{x}=e_{p 1} x_{1}+e_{p 2} x_{2}+\ldots+e_{p p} x_{p}
\end{aligned}
$$

$P C_{1}=$ the $1^{\text {st }} \mathrm{PC}$ from linear combination, the $1^{\text {st }}$ biggest variance

$P C_{2}=$ the $2^{\text {nd }} P C$ from linear combination, the $2^{\text {nd }}$ biggest variance

$P C_{p}=$ the $p^{\text {th }} \mathrm{PC}$ from linear combination, the $p^{\text {th }}$ biggest variance

$x_{1}=$ the $1^{\text {st }}$ origin random variable

$x_{2}=$ the $2^{\text {nd }}$ origin random variable :

$x_{p}=$ the $p^{\text {th }}$ origin random variable

$\mathbf{e}_{p}=$ the $p^{\text {th }}$ eigenvector

The $i^{\text {th }}$ PC from combination linear can be generally written as follows in (2).

$P C_{i}=\mathbf{e}_{i}^{\prime} \mathbf{x}$

So that, $\quad \operatorname{Var}\left(P C_{i}\right)=\mathbf{e}_{i}^{\prime} \mathbf{\Sigma} \mathbf{e}_{i}=\lambda_{i}, i=1,2, \ldots, p \quad$ and $\operatorname{Cov}\left(P C_{i}, P C_{m}\right)=\mathbf{e}_{i}^{\prime} \mathbf{\Sigma} \mathbf{e}_{m}=0, i \neq m . \quad$ The principal components do not have any correlation among each of them and have the same variance with the eigenvalue of $\Sigma$, so as in (3).

$$
\begin{aligned}
& \sum_{i=1}^{p} \operatorname{Var}\left(x_{i}\right)=\sigma_{11}+\sigma_{22}+\ldots+\sigma_{p p} \\
& \sum_{i=1}^{p} \operatorname{Var}\left(\mathrm{PC}_{i}\right)=\lambda_{1}+\lambda_{2}+\ldots+\lambda_{p}
\end{aligned}
$$

The number of principal components is $m$ where $m<$ $p$ and the proportion of total variance that can be explained by the $m^{\text {th }}$ principal component as follows:

Variance Proportion for

$$
m^{\text {th }} \mathrm{PC}=\frac{\lambda_{m}}{\lambda_{1}+\lambda_{2}+\ldots+\lambda_{p}}
$$

There are several references to determine the number of PC, including:

1. Observing the screen plot pattern that illustrates the amount of the eigenvalue. The number of PC chosen is determined by the largest range between points where the PC selection process will be terminated if the range between points is lower (visible slope). However, this approach is more subjective, so we need another approach that is more objective.

2. PC selection is based on the number of eigenvalues greater than one, which represents a high level of homogeneity between variables.

3. The selected PCs are expected to provide a cumulative percentage variance of minimum $80 \%$ (Johnson and Wichern, 2007).

\section{Model Output Statistics (MOS) Modeling using Ridge Regression}

Model Output Statistics (MOS) is a modeling between the weather observation results and the output of NWP based on regression. The general mathematical model of MOS is shown in (5) (Wilks, 2007).

$\hat{Y}_{i}=f_{\text {MOS }}\left(X_{i}\right)$.

$\hat{Y}_{i}=$ weather forecast at the time- $t$

$X_{i}=$ output variables of NWP at the tine- $t$.

Ridge regression is one of methods used to reduce the influence of correlated predictors (Rencher and Schaalje, 2007). This multicollinearity can lead to singularity in the $\mathbf{X}^{\prime} \mathbf{X}$ matrix and causes the estimation of the regression parameters in (6) to be invalid.

$\hat{\boldsymbol{\beta}}=\left(\mathbf{X}^{\prime} \mathbf{X}\right)^{-1} \mathbf{X}^{\prime} \mathbf{y}$.

Ridge regression uses a non-negative constant $\lambda$ that modifies equation (6) to calculate a more efficient regression coefficient. Equation (7) is an equation commonly used to estimate ridge regression coefficient.

$\hat{\boldsymbol{\beta}}_{\text {ridge }}=\left(\mathbf{X}^{\prime} \mathbf{X}+\lambda \mathbf{I}\right)^{-1} \mathbf{X}^{\prime} \mathbf{y}$

$\lambda$ constants can be chosen intuitively (by default) or by cross-validation techniques based on the lowest Root Mean Square Error (RMSE). The higher $\lambda$ causes the $\beta_{p}$ coefficient gets closer to 0 or causes the $p$ parameter to have less effect on the response.

\section{Model Validation}

One of the indicators used to evaluate the quality of weather forecasts is the Root Mean Square Error of Prediction (RMSEP). RMSEP is the square root of MSE, which is the average number of squares of the difference between forecast and observation values. Equation (8) is a formula for calculating RMSE.

$\mathrm{RMSEP}=\sqrt{\frac{1}{n} \sum_{i=1}^{n}\left(y_{i}-\hat{y}_{i}\right)^{2}}$ 
The smaller the RMSEP value, the better the forecasting model. The RMSEP value can be used as a base for model validation which is shown in Table 1 .

Table 1. RMSEP Value Criteria for Temperature (Source: BMKG).

\begin{tabular}{cc}
\hline Criterion & RMSEP \\
\hline Very good & $0.0-0.4$ \\
Good & $0.5-0.8$ \\
Intermediate & $0.9-1.2$ \\
Bad & $1.3-1.6$ \\
Very bad & $>1.6$ \\
\hline
\end{tabular}

\section{Bias Corrector Measurement}

The percentage increase in the MOS model with respect to NWP is shown by percentage Improval (\% IM) that can be calculated using the formula as (9)

$$
\% I M=\frac{R M S E P_{N W P}-R M S E_{M O S}}{R M S E_{N W P}} \times 100 \% \text {. }
$$

The value of $\% \mathrm{IM}$ is from $0 \%$ to $100 \%$. The higher value \%IM means the MOS model has a better correction of NWP's biased forecasting result (Sutikno et al., 2019).

\section{Data and Variables}

The data used in this study are the secondary data from Meteorology, Climatology and Geophysics Agency (BMKG), i.e. the data of CCAM (conformal cubic atmospheric model) NWP data from 1st of January 2009 to 31st of December 2010 or 708 days. The location of research focus is meteorological station, i.e. Citeko, Curug, Dermaga, and Cengkareng. The response variable is the surface's weather observation data that consist of Tmax and Tmin measured directly in every station. The predictor variable is the output of the NWP CCAM model. Meanwhile, the NWP CCAM parameter used is taken from the previous study's parameter by a meteorologist, shown in Table 2 for the MOS model.

Table 2. NWP CCAM Parameters.

\begin{tabular}{llll}
\hline No & NWP Parameter (code) & Level & Unit \\
\hline 1 & Surface Pressure Tendency (dpsdt) & surface & $\mathrm{hPa}$ \\
2 & Water Mixing Ratio (mixr) & $1,2,4$ & $\mathrm{~g} / \mathrm{kg}$ \\
3 & Vertical Velocity (omega) & $1,2,4$ & $\mathrm{knot}$ \\
4 & PBL depth (pblh) & surface & $\mathrm{meter}$ \\
5 & Surface Pressure (ps) & surface & $\mathrm{hPa}$ \\
6 & Mean Sea Level Pressure (psl) & surface & $\mathrm{hPa}$ \\
7 & Screen Mixing Ratio (qgscm) & surface & $\mathrm{g} / \mathrm{kg}$ \\
8 & Relative Humidity (rh) & $1,2,4$ & $\%$ \\
9 & Precipitation $(\mathrm{rnd})$ & surface & $\mathrm{Mm}$ \\
10 & Temperature & $1,2,4$ & $\mathrm{Celcius}$ \\
11 & Maximum Screen Temperature (tmaxcr) & surface & $\mathrm{Celcius}$ \\
12 & Minimum Screen Temperature (tmincr) & surface & $\mathrm{Celcius}$ \\
13 & Pan Temperature (tpan) & surface & $\mathrm{Celcius}$ \\
14 & Screen Temperature (tscrn) & surface & $\mathrm{Celcius}$ \\
15 & Zonal Wind $(\mathrm{u})$ & $1,2,4$ & $\mathrm{knot}$ \\
16 & Friction Velocity (ustar) & surface & $\mathrm{m} / \mathrm{sec}$ \\
17 & Meridional Wind $(\mathrm{v})$ & $1,2,4$ & $\mathrm{knot}$ \\
18 & Geopotential Height $(\mathrm{zg})$ & $1,2,4$ & $\mathrm{~meter}$ \\
\hline
\end{tabular}

Besides the 7 parameters that are measured in the different pressure levels, 11 other parameters were measured only in the surface level with a height of \pm 2 meters above sea level. Hence, the number of NWP parameters is 32 parameters. Then, each of the 32 parameters is measured on the nine grid $(3 \times$ x 3$)$ measurements, so there are 288 parameters in the total.

\section{RESULTS AND DISCUSSION}

Analysis and evaluation steps for Dermaga Station will be explained in detail, while other stations are few summary because of the analysis step actually the same.

\section{Pre-Processing the NWP Data using PCA Method}

PCA used to reduce the complexity of model which there are $288 \mathrm{NWP}$ variables in the total. The number of principal components is determined by choosing which have an eigenvalue larger than 1. The principal component for NWP variable in Dermaga station is shown in Table 3.

Table 3. The Number of PC and Eigenvalue in Dermaga Station.

\begin{tabular}{llll}
\hline Variable & The Number of PC & Eigenvalue & Var. \\
\hline Dpsdt & 1 & 8,998 & $99,98 \%$ \\
mixr1 & 1 & 7,632 & $84,80 \%$ \\
mixr2 & 1 & 8,367 & $92,96 \%$ \\
mixr4 & 1 & 8,618 & $95,76 \%$ \\
omega1 & 2 & 5,$832 ; 2,139$ & $88,56 \%$ \\
omega2 & 2 & 5,$597 ; 2,266$ & $87,37 \%$ \\
omega4 & 2 & 6,$299 ; 1,273$ & $84,13 \%$ \\
Pblh & 1 & 7,483 & $83,15 \%$ \\
Ps & 1 & 8,529 & $94,77 \%$ \\
ps1 & 1 & 8,995 & $99,95 \%$ \\
qgscrn & 2 & 6,$651 ; 1,104$ & $86,17 \%$ \\
rh1 & 2 & 7,$186 ; 1,182$ & $92,98 \%$ \\
rh2 & 1 & 8,253 & $91,70 \%$ \\
rh4 & 1 & 8,591 & $95,45 \%$ \\
rnd & 1 & 7,359 & $81,76 \%$ \\
temp1 & 1 & 7,943 & $88,25 \%$ \\
temp2 & 1 & 8,381 & $93,13 \%$ \\
temp4 & 1 & 8,753 & $97,26 \%$ \\
tmaxscr & 1 & 8,640 & $96,00 \%$ \\
tminscr & 1 & 7,542 & $83,80 \%$ \\
tpan & 1 & 7,975 & $88,61 \%$ \\
tscrn & 1 & 7,984 & $88,72 \%$ \\
u1 & 1 & 7,507 & $83,41 \%$ \\
u2 & 1 & 8,012 & $89,02 \%$ \\
u4 & 1 & 8,877 & $98,63 \%$ \\
ustar & 2 & 6,$014 ; 1,393$ & $82,29 \%$ \\
v1 & 2 & 6,$371 ; 1,482$ & $87,25 \%$ \\
v2 & 2 & 6,$850 ; 1,136$ & $88,74 \%$ \\
v4 & 1 & 8,607 & $95,64 \%$ \\
zg1 & 1 & 8,780 & $97,55 \%$ \\
zg2 & 1 & 7,910 & $87,89 \%$ \\
zg4 & 2 & 5,$057 ; 3,814$ & $98,57 \%$ \\
\hline & & & \\
\hline
\end{tabular}


Table 3 shows that 1 component is very good at representing several NWP parameters at the Pier station. NWP parameters, represented by 2 components, are given all omega and ustar parameters. Dimension reduction using PCA resulted in 41 components from 32 parameters measured in 9 grids.

The variability of NWP parameter that can be explained by the principal components varies from $81.76 \%$ until almost $100 \%$. This proves that the correlation between the grids in the NWP parameter is relatively high, so that the dimension reduction is an appropriate step to correct the bias of NWP output. Furthermore, the principal components formed from all NWP parameters are used as predictor variables in the MOS modeling using ridge regression.

\section{Forecast Modeling of Tmax and Tmin using Ridge Regression}

Ridge regression uses the constant $\lambda$ to overcome the $\mathbf{X}^{\prime} \mathbf{X}$ matrix singularity. The constant $\lambda$ is expected not too large. This is to avoid the ridge regression coefficient that is getting closer to 0 . If it happens, then the estimated value of the response is also close to 0 . While the air temperature in Indonesia almost never occurs in the range of $0^{\circ} \mathrm{C}-10^{\circ} \mathrm{C}$. Figure 1 helps visual determination of $\lambda$ constant.

(a)

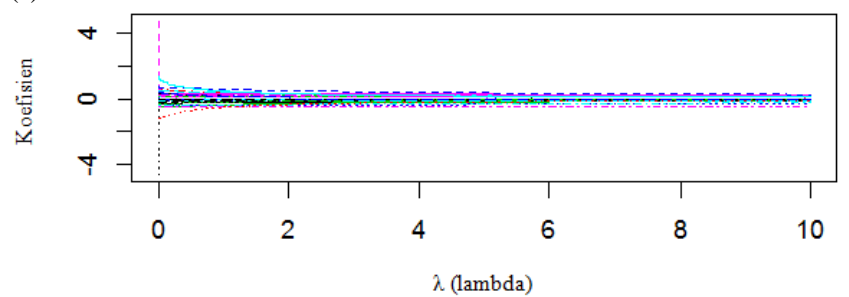

(b)

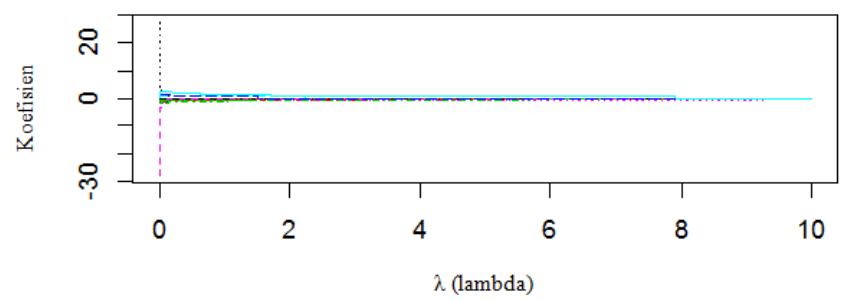

Figure 1. Ridge Regression Coefficient Convergence (a) maximum temperature; (b) minimum temperature.

The determination of convergence based on Figure 1 should be avoided because it is subjective. However, in this case, the constant $\lambda$ even remains non-converging when $\lambda$ approaches 100 , so it was decided to use visual aids in determining $\lambda$. According to Draper and Smith (1998), the lower and upper limits for $\lambda$ is not strictly determined. However, the upper limit should not be greater than 10 or 20 to avoid a meaningless regression coefficient because it is close to 0 . Figure 1 indicate that the regression coefficients for Tmax and Tmin converge when $\lambda$ is 9 or more. Table 4 is the Ridge regression coefficient for Dermaga station with $\lambda=9$.

Table 4. Ridge Regression Coefficient for Dermaga Station.

\begin{tabular}{|c|c|c|}
\hline Predictor & Tmax & Tmin \\
\hline PC.dpsdt & $-0,075$ & $-0,099$ \\
\hline PC.mixr1 & 0,123 & $-0,125$ \\
\hline PC.mixr2 & 0,102 & $-0,129$ \\
\hline PC.mixr4 & 0,030 & 0,008 \\
\hline PC1.omega1 & 0,153 & 0,165 \\
\hline PC2.omega1 & 0,212 & 0,015 \\
\hline PC1.omega2 & $-0,099$ & $-0,051$ \\
\hline PC2.omega2 & $-0,029$ & $-0,038$ \\
\hline PC1.omega4 & $-0,017$ & 0,083 \\
\hline PC2.omega4 & $-0,082$ & 0,031 \\
\hline PC.pblh & $-0,041$ & 0,010 \\
\hline PC.ps & 0,024 & 0,037 \\
\hline PC.psl & 0,027 & 0,066 \\
\hline PC1.qgscrn & $-0,005$ & $-0,153$ \\
\hline PC2.qgscrn & $-0,067$ & 0,054 \\
\hline PC1.rh1 & 0,027 & $-0,021$ \\
\hline PC2.rh1 & $-0,069$ & $-0,172$ \\
\hline PC.rh2 & 0,031 & $-0,261$ \\
\hline PC.rh4 & 0,037 & 0,009 \\
\hline PC.rnd & 0,071 & $-0,022$ \\
\hline PC.temp1 & $-0,108$ & $-0,083$ \\
\hline PC.temp2 & 0,256 & 0,066 \\
\hline PC.temp4 & 0,008 & $-0,219$ \\
\hline PC.tmaxscr & $-0,513$ & 0,149 \\
\hline PC.tminscr & $-0,108$ & $-0,084$ \\
\hline PC.tpan & 0,053 & $-0,026$ \\
\hline PC.tscrn & $-0,099$ & $-0,010$ \\
\hline PC.u1 & $-0,301$ & 0,055 \\
\hline PC.u2 & $-0,236$ & 0,016 \\
\hline PC.u4 & 0,141 & 0,124 \\
\hline PC1.ustar & 0,048 & 0,014 \\
\hline PC2.ustar & 0,007 & $-0,088$ \\
\hline PC1.v1 & $-0,012$ & $-0,075$ \\
\hline PC2.v1 & 0,012 & $-0,049$ \\
\hline PC1.v2 & $-0,094$ & 0,010 \\
\hline PC2.v2 & 0,089 & 0,053 \\
\hline PC.v4 & $-0,032$ & $-0,021$ \\
\hline PC.zg1 & $-0,096$ & 0,004 \\
\hline PC.zg2 & 0,022 & $-0,166$ \\
\hline PC1.zg4 & 0,001 & 0,022 \\
\hline PC2.zg4 & 0,079 & 0,290 \\
\hline
\end{tabular}

After the regression coefficient is obtained, the next step is to form a model based on the regression coefficients in Table 4. The weather forecast model of Tmax and Tmin using the ridge regression at Dermaga Station is presented in Table 5 . 
Table 5. Ridge Regression Model for Weather Forecast in Dermaga Station.

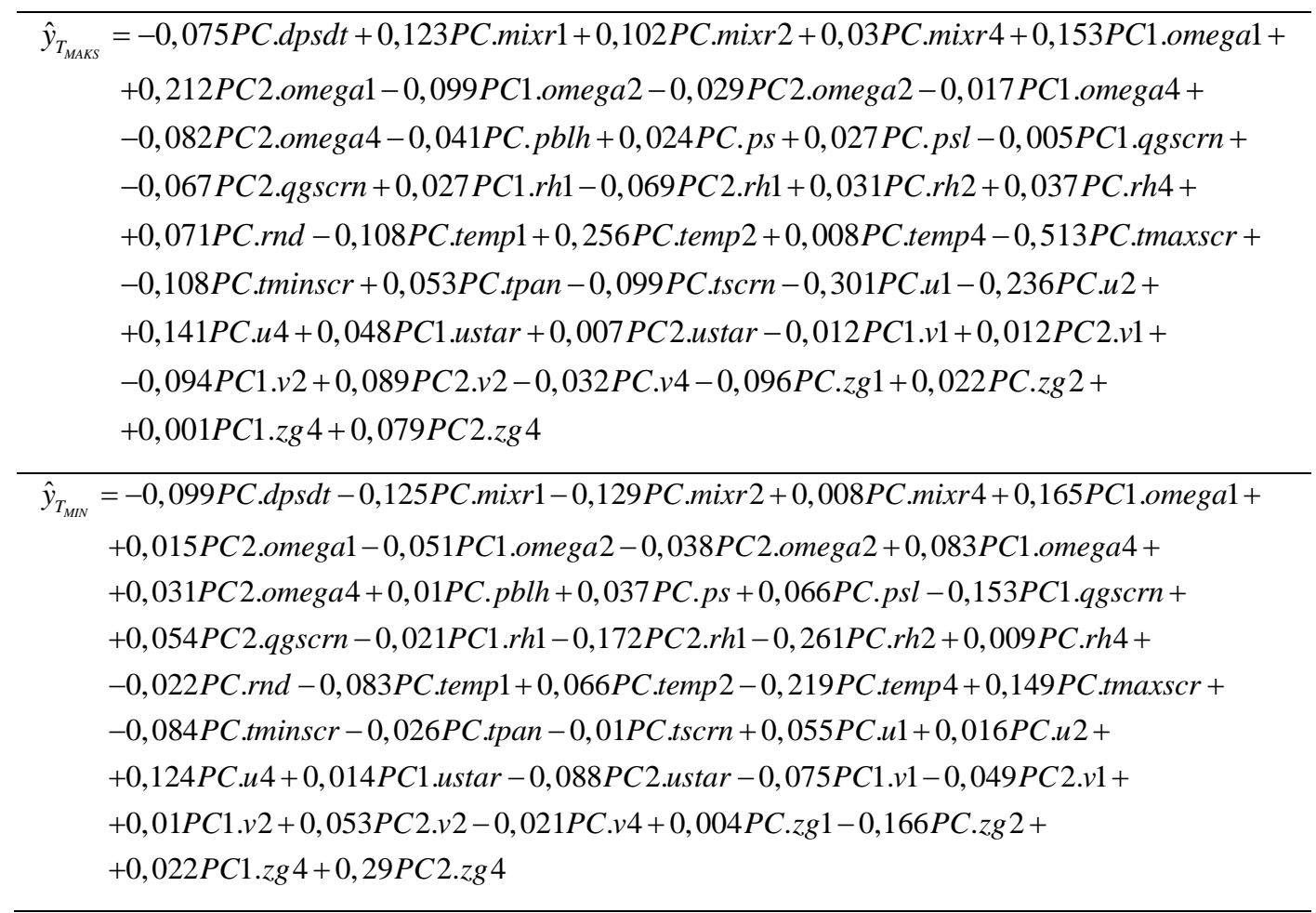

Based on the ridge regression model in Table 5, the weather forecast results obtained at the Dermaga Station are briefly presented in Table 6 .

Table 6. The Temperature Forecast Results at the Dermaga Station.

\begin{tabular}{lllll}
\hline Date & $\begin{array}{l}\text { Tmax } \\
\text { Obs. }\left({ }^{\circ} \mathbf{C}\right)\end{array}$ & $\begin{array}{l}\text { Tmax } \\
\text { Ridge } \\
\left({ }^{\circ} \mathbf{C}\right)\end{array}$ & $\begin{array}{l}\text { Tmin } \\
\text { Obs. }\left({ }^{\circ} \mathbf{C}\right)\end{array}$ & $\begin{array}{l}\text { Tmin } \\
\text { Ridge } \\
\left({ }^{\circ} \mathbf{C}\right)\end{array}$ \\
\hline $1 / 1 / 2009$ & 30.1 & 30.83 & 23.2 & 22.39 \\
$1 / 2 / 2009$ & 32.1 & 31.80 & 22.3 & 22.44 \\
$1 / 3 / 2009$ & 31.5 & 31.85 & 21.2 & 21.99 \\
$1 / 4 / 2009$ & 33 & 32.14 & 20.4 & 21.44 \\
$1 / 5 / 2009$ & 32 & 32.12 & 19.8 & 22.02 \\
$: \cdot$ & $\vdots$ & $\vdots$ & $\vdots$ & $\vdots$ \\
$12 / 27 / 2010$ & 29.1 & 29.15 & 23.2 & 22.42 \\
$12 / 28 / 2010$ & 30.9 & 29.68 & 22.8 & 23.22 \\
$12 / 29 / 2010$ & 30 & 31.14 & 22 & 22.93 \\
$12 / 30 / 2010$ & 30 & 31.87 & 23.8 & 22.99 \\
$12 / 31 / 2010$ & 30.6 & 30.60 & 23.4 & 22.93 \\
\hline
\end{tabular}

\section{Model Validation}

Model validation aims to determine the accuracy and goodness of the model formed. Validation of the ridge regression model is done by testing the forecast with the observation data, so that the RMSEP value is obtained. The RMSEP values at the 4 stations are shown in Table 7.
Table 7. RMSEP Values for Ridge Regression in 4 Stations.

\begin{tabular}{llll}
\hline Station & Variable & RMSEP & RMSEP Criterion \\
\hline \multirow{2}{*}{ Cengkareng } & Tmax & 0.9771 & Intermediate \\
& Tmin & 0.7236 & Good \\
\hline \multirow{2}{*}{ Citeko } & Tmax & 1.0785 & Intermediate \\
& Tmin & 0.6363 & Good \\
\hline \multirow{2}{*}{ Curug } & Tmax & 1.0846 & Intermediate \\
& Tmin & 0.6540 & Good \\
\hline \multirow{2}{*}{ Dermaga } & Tmax & 0.9449 & Intermediate \\
& Tmin & 0.7746 & Good \\
\hline
\end{tabular}

The RMSEP value of the Tmax modeling using ridge regression has an intermediate result according to the BMKG criterion. In the other side, the RMSEP value of the Tmin has a good result. The result of this ridge regression modeling is then regarded as the MOS model.

\section{Comparison of Accuracy between NWP and MOS Result}

NWP model produces biased predictions that require post-processing using the MOS method, i.e. ridge regression. Percentage Improval (\%IM) indicates the amount of NWP bias that can be corrected by MOS. RMSE $_{N W P}$ is obtained by comparing NWP data on the fifth grid (the closest grid to the observation station) and observation data. The amount of NWP bias that can be corrected by ridge regression as MOS at 4 stations is shown in Table 8. 
Table 8. The Value of RMSE ${ }_{N w P}, R_{M S E}$ Mos, and \%IM.

\begin{tabular}{lllll}
\hline Station & Variable & RMSEP$_{\text {MOS }}$ & RMSEP $_{\text {NWP }}$ & \%IM \\
\hline \multirow{2}{*}{ Cengkareng } & Tmax & 0.9771 & 6.0588 & 83.8725 \\
& Tmin & 0.7236 & 1.1713 & 38.2268 \\
\hline \multirow{2}{*}{ Citeko } & Tmax & 1.0785 & 11.3443 & 90.4928 \\
& Tmin & 0.6363 & 5.3484 & 88.1023 \\
\hline \multirow{2}{*}{ Curug } & Tmax & 1.0846 & 6.6605 & 83.7159 \\
& Tmin & 0.6540 & 0.9728 & 32.7774 \\
\hline \multirow{2}{*}{ Dermaga } & Tmax & 0.9449 & 7.2993 & 87.0554 \\
& Tmin & 0.7746 & 1.6533 & 53.1479 \\
\hline
\end{tabular}

Table 8 shows that the RMSEP obtained from the NWP model is consistently greater than the RMSEP of the MOS model. It means that the MOS model is better used to predict Tmax and Tmin rather than the NWP model. The MOS model is able to correct from $32.7774 \%$ until $90.4928 \%$ of the NWP which is biased to predict Tmax and Tmin. Table 8 also shows that the RMSEP $_{\text {NwP }}$ at Citeko Station is the largest among the four other stations so that the Citeko Station has the \% IM which holds the largest bias corrector. This is because the Citeko Station is located in a mountainous area which has complex vegetation, thus generating a large bias for the NWP model.

\section{CONCLUSION}

Most of the principal components that are formed by the result of the NWP parameter reduction which is measured in 9 grids are one component. Those principal components have explained until almost $100 \%$ variability of NWP parameter. The validation result of the ridge regression with the RMSEP shows that the Tmax is included in the intermediate criteria for all stations and Tmin is included in the good criteria for all stations. The prediction results from the ridge regression are more accurate than the NWP model and are able to correct up to $90.49 \%$ of the biased NWP for Tmax forecasting. Hence, we can conclude that the ridge regression as MOS can solve the NWP problem regarding the dimension reduction and forecasting. The modeling result from this study is recommended to be used by BMKG in forecasting the temperature because this model is capable of producing a smaller bias compared to the NWP model from the BMKG.

\section{ACKNOWLEDGEMENTS}

The entire data used in this study were supported by the Meteorology, Climatology and Geophysics Agency (BMKG) of Indonesia. The fund for this study is supported by the Ministry of Research, Technology and Higher Education of Indonesia for the grant of the National Strategic Research 2018.

\section{REFERENCES}

BMKG. 2006. Uji Operasional dan Validasi Model Output Statistik (MOS). Jakarta: BMKG

Draper, N. R. and Smith, H. 1998. Applied Regression Analysis Third Edition. New York: John Wiley \& Sons, Inc.

Glahn, H. R. and Lowry, D. A. 1972. The Use of Model Output Statistics (MOS) in Objective Weather Forecasting. Journal of Applied Meteorology. pp. 1203-1211.

Johnson, R. A. and Wichern, D. W. 2007. Applied Multivariate Statistical Analysis Sixth Edition. New Jersey: Prentice Hall.

Rencher, A. C. and Schaalje, G. B. 2007. Linear Models in Statistics Second Edition. New Jersey: John Wiley \& Sons, Inc.

Sutikno, Nisaa', Z. and Nur 'Anisa', K. 2019. Temperature and Humidity Forecast via Univariate Partial Least Square and Principal Component Analysis. Malaysian Journal of Science. pp. 1-13.

Wilks, D. S. 2007. Statistical Methods in the Atmospheric Sciences Second Edition, Meteorological Applications. London: Elsevier. 